\section{A Maper}

$$
\text { ox }
$$

\section{SYPHILIS OF THE HEART AND AORTA.* BY}

JOHN COWAX, M.D., AND J. STEVEN FAULDS, M.B. (From the Royal Infirmary, Glasgow.)

Ir is extremely difficult to assess the importance of syphilis as a cause of heart disease. We have little knowledge of its incidence among the general population, and still less of its influence upon the heart. In consequence we do not know whether the cases which we recognize as syphilitic lieart disease are merely isolated curiosities or the common sequel of uncured syphilis.

The immediate mortality of syphilis is trifling; it kills through risceral complications. But no one indicates the specifie nature of the organic disease upon the death certificate, and cirrhosis of the liver, cerebral disease, etc., figure on the records. The mortality of syphilis must, however, be considerable, for aneurysm alone kills a thousand people every year in England and Wales, and the rast majority of fatal aneurysms are due to syphilis.

The disease is common. Osler and Macrae ${ }^{1}$ state that it has been estimated that 12 per cent. of the adult population in Berlin and 15 per cent. in Paris are affected, while in America the incidence is said to vary between 6 and 40 per cent., a positive Wassermann reaction being present in 15 per cent. of hospital patients. Though in England statistics are less numerous, it is known that about 100,000 men of our armies in the last war were infected, ${ }^{2}$ and " as it is rensonable to suppose that more venereal disease was imported into France than vice versa," the incidence at home must be considerable. In our wards routine Wassermann tests showed positive results in 8.6 per cent. of the male, and 7.2 per cent. of the female patients, quite irrespective of their ailments.

The influence of syphilis in heart disease is evidently considerable. Carey Coombs ${ }^{3}$ states that syphilis was present in 9 per cent. of his hospital, and 4 per cent. of his private, patients. Allan states that a syphilitic infection was present in 63 of 320 cases of "failing heart" admitted into hospital (19.7 per cent.). Our own figures, in a series of 395 cases of organic valvular disease, show a syphilitic incidence of $\mathbf{1 7 . 7}$ per cent. Routine Wassermann tests have shown that the incidence is much greater than was formerly supposed.

The eridence from the pathological side suggests that tha higher figures are the more accurate. In our series of 390 cases presenting naked-ere signs of cardio-vaseular disease 60 (15.3 per cent.) were syphilitic. It must be remembered that these figures are a conservative estimate, for a negative Wassermann reaction does not prove that syphilis has not been active in the past, and the results or srphilis cannot always be recognized as syphilitic after death. Any part of the heurt may be affected, and any disease of the heart may be due to syphilis. In some diseases syphilis is the common cause, in others the exception.

The Pathologicil Lenions.

The chief pathological lesions are: endarteritis obliterans, aortitis, aortic valvular disease, gumma of the heart, and myocardial fibrosis. The other lesions are rarely syphilitic. We have analysed a series of 1,000 consecutive necropsies. Of these cases 390 presented some disease of the heart or aorta, judged by naked-eye examination. These lesions wore not, however, necessarily the cause of death, some of the patients having died from preumonia, cancer, etc. Of these 380 cases 60 presented some eridence of syphilitic heart disease.

Endarteritis Obliterans.

Fndarteritis obliterans occurs chiefly in the smaller arteries and the arterioles. It is always local in its distribution, and never affects more than a short length of a ressel, but, as it affects the whole circumference of the * Read in opening a cliscussion in the Section of Medicine at the
Annual Meeting of the British Medical Association, Manohester, 1829. wall, the lumen is always narrowed, and not infrequently completely blocked, usually by thrombosis.

In this lesion the intima is always thickened, the newformed connective tissue being in the early stage loose and cellular, with much hyperplasia of the elastic clements. In the later stages it is poorly nucleated and hyaline, with degeneration of the elastic tissue. The media may be little altered or greatly thinned. The alventitia is always thickened, sometimes to an extraordinary degree, and, like the intima, shows new formation of elastic tissue. The relative thickness of the intima and the adventitia varies considerably in different cases, sometimes the one and sometimes the other showing the maximal change. Gross calcareous and fatty changes are rarely found.

Muirs states that this lesion represents the ordinary reaction of the arterial wall to an irritant, which often approaches it from without. It is common in the ricinity of an ulcerative process, in pulmonary silicosis, tuberculous lesions, and granular kidneys. It is perhaps the special arterial lesion in syphilis, and occurs at all stages of the disease, in the chancre as well as in the tertiary lesions. It is always present in gummata, and occurs as well in their absence. Its accurrence in the adventitia of tho aorta, and in the arteries of the heart, plays an important part in the causation of aneurysm and of ischaemic fibrosis of the myocardium.

Syphilitic Aortitis.
The syphilitic lesions in the aorta oceur as soft elernted plaques, usually sharply circumscribed, and of considerable thiekness. They have a greyish, gelatinous appearance, and as they extend and fuse may affect considerable tracts. In older specimens, where absorption and contraction have ensued, the plaques are thin and pearly white; the ultimate. cicatrix has sometimes a stellate appearance. The thoracic aorta, in particular its first part, is most often involved, and this selection is unfortunate, as a spread downwards is likely to involve the orifioes of the coronary arteries or the aortic cusps, with resultant ischacmia of the cardiac muscle or aortic valvular disease. The aortic wall, too, is always weakened, and syphilitic aortitis is thus the common cause of aortic aneurysm.

The early lesions in the aorta are, howerer, not intimal but adventitial. The earliest change consists of an infiltration into the outer coat, accompanied by endarteritis and periarteritis. The infiltration then extends along the vessels into the media, branching ont in places into irregular collular areas in which there is also new formation of thin-walled vessels. In some cases necrosis of the media ensues. This leads to absorption of the muscular and elastic tissues of the media, and sections stained to show the elastic tissue present breaks, or "windows," corresponding to the cellular areas, which may extend across even the full extent of the media. At a later period new formation of connective tissue takes place, with resultant fibrous patches in the media, wholly devoid of muscle and elastic tissue. This scar tissue is weak, and localized depressions or pouches, the potential predecessors of aneurysm, are often visible on the intimal aspect.

If the aorta is affected immediately above the valve the aortic cusps may be affected, so producing valvular disease. The orifices of the coronary arteries, too, may become involved, with narrowing, or eren complete occlusion, of the lumen. Occasionally a plaque may form on the mitral valve, but it is usually small, and syphilis plays an insignificant part in the production of chronic mitral disease.

The essentinl cause of ancurysm is weakness of the arterial wall, which gives way before the pressure of the blood within the vessel; this woakness mainly affects the media. Medial weakness may arise in different ways. It is most commonly due to syphilis, but a comparable lesion may occur from an acute infection-for instance in phthisis-if an ulcerative process happens to hit an artery; or in ulceration of the stomach. Thus fatal gastric haemorrhage may arise from the rupture of an aneurysm of the splenic artery, which has become involved in a chronic ulcer of the stomach. The media: may also be affected from within in acute aortitis secondary to acute endocarditis or sepsis elsewhere. It may be damaged by the pressure of an atheromatous patch to \3580, 
a degree that permits dilatation; trauma may be an occasional cause. But aneurysm is most commonly of syphilitic origin.

\section{Chronic Aortic Valvular Disease.}

There is considerable difficulty in determining the precise causes of chronic valvular disease. More than one cause is often active; tho chronicity of the disease prevents accurate observation of its progress, and the effects of different causes aro similar, the ultimate result depending upon the site of the lesion rather than on the nature of the cause. In each case the cause has usually to be deduced from the history of the development of the symptoms, the antecedent illnesses, and the associated lesions in the aorta and elsewhere, rather than from the actual lesions which are found to be present.

Varied lesions are found at the post-mortem examination. The cusps are thickened, hardened, and opaque, the change being sometimes uniformly distributed throughout them, and sometimes confined to, or most extreme at, the free margins. Calcareous and atheromatous deposits are not uncommon, and, though usually merely an addition to a general lesion, are sometimes an isolated lesion in a fairly normal cusp. The calcareous masses may be large, and from their bulk interfere with the proper action of the valve.

The cusps may bo shortened, or adherent to each other. Shortening produces incompetence, and adhesion narrowing, of the orifice; the two conditions frequently coexist. The ultimate result varies. The cusps may be more or less uniformly adherent, forming a funnel or dome or diaphragm. The orifice may be greatly narrowed, and rounded, linear, or stellate. Not infrequently warty outgrowths of varying size and consistence occur near the free margins. In other cases there is loss of substance, and the edges are puckered and uneven. The line of attachment of a cusp may be destroyed, and a part of it may be freely mobile in the blood stream, or, if calcified, project stiffly into the lumen.

A cusp may be perforated through its substance. This is most commonly the sequel of acute endocarditis, but, rarely, it follows severe muscular exertion or an external trauma, and then usually in a valve which has already been damaged. In the majority of chronic lesions the edges of the cusps are inverted to some extent. In exceptional cases the cusps are voluminous and soft, with everted edges, thus producing gross incompetence of the valve.

Chronic valvular disease may be of congenital origin, or it may follow acuto endocarditis, a syphilitic infection, or mechanical strain; it may be merely an incident in a general cardio-vascular degeneration. Aortio valves with thick uneven edges and adhesion of the cusps, those which present scars in the immediate vicinity of the valve, whether in the aorta or the adjacent endocardium, and those accompanied by chronio disease of the mitral valve, are most frequently the result of acute endocarditis, usually of rheumatic origin. Cases which present syphilitic masses or scars on the proximal parts of the aorta, with comparable lesions on the aortic valves, are evidently specific in origin. But in many cases atheromatous disease is also present, and the exact origin of the valvular lesion is, in consequence, difficult to determine.

\section{Atheroma.}

Atheroma is by far the most common affection of the vascular tree. It affects arteries of all sizes, from the smallest to the largest, and is most common in the first part of the aorta, the coronary arteries, the cerebral arteries, and those of the limbs. The lesions may be widespread or localized to some particular set. of vessels. A chronic affection of the aortio valve, with thickening and induration, often coexists, from a spread on to the valve of the samo process that has affected the arteries.

The causes of atheroma are numerous. It is most common in persons of advanced years, representing the scars and bruises of ancient wars and the defective nutrition of a failing organism. In some instances the special localization points to stress or strain as causal, at any rate in part; in others, toxic influences or metabolic disturbances seem to have been active. In the majority, of cases many or all of these factors havo been active in the past. Atheroma is especially frequent in cases of chronic renal disease, with a high blood pressure and thickened arteries. If, in addition, a rheumatio or a syphilitic infection has been antecedent, the causes of the ultimate disease must of necessity be obscure. The exact influence of each can only bo determined by the survey of a large series of cases.

\section{Myocardial Disease.}

There are several specific myocardial lesions, but in the majority of cases the myocardial lesions are only side issues, the result of coronary disease, and so in no way specific. The typical specific lesion is the gumma, but its occurrence is rare, and none is recorded in our series. Gummata vary in size from microscopic lesions to tumours as large as a pigeon's egg. They may be single or multiple; they may. occur anywhere; and may occasion disturbances of the cardiac rhythm if they involve the primitive tissues. A diffuse fibrosis (subacute interstitial fibrosis) has been found during the secondary stages in the adult, and, more often, in congenital syphilis in infants and young childreri.

The most important, and the most frequent, sequel of syphilis follows disease of the coronary arteries and interference with the blood supply of the muscle. The arteries may be occluded at their orifice by a syphilitic plaque in the aorta, or narrowed in their course by a specific endarteritis. If the circulation is completely blocked an infarct occurs, while if the narrowing is incomplete a para-arterial fibrosis results. The ultimate lesions in the muscle are not syphilitic, though they have a syphilitic cause.

\section{Pericarditis.}

The influence of syphilis as a cause of pericarditis is negligible. Pericarditis may, of course, occur in relation to a gumma of the heart or a diffuse syphilitic fibrosis. Syphilis seems sometimes to be the cause of indurativo mediastino-pericarditis, the inflammation usually arising beneath the diaphragm, about the liver, and spreading upwards. But the few cases of syphilitic pericarditis which have been reported are unconvincing, and can be neglected.

\section{The Clinical Data. \\ Aortitis.}

Aortitis may occur in any acute infection, but it is rare save in acute endocarditis, particularly of the aortic valve. The infection may be conveyed directly, by continuity or through the aortic blood, or through the vasa in the adventitia. The most common organisms are streptococci; but any pathogenic bacteria may be active. It occurs not infrequently in acute rheumatism and rarely in tuberculosis, but the lesions in these diseases are usually limited in their extent and not excessive in degree.

The chief cause of aortitis is undoubtedly syphilis. It is difficult to say when the lesions arise, but it seems probable that they may occur early, in untreated cases even in the secondary stages, though they are rarely recognized until many years have passed. The reason of this failure to recognize its presence is clear. Aortitis per se does not occasion any symptoms. These only arise if " something happens" -an aneurysm forming, or the aortic cusps or the orifices of the coronary arteries becoming implicated in the syphilitic process. Aortitis is thus most commonly a post-mortem discovery. It was present in 59 of the 60 syphilitic cases in the post-morten series. Of these, 29 died from lesions in the heart and aorta, the remaining 30 from other causes, the most frequent of which were cerebral.

In this form of syphilitio disease it is impossible to ascribe particular symptoms to particular lesions, for aneurysm, aortic valvular disease, and obstruction of the coronary arteries are all almost always present in some degree in every case. The degree, of course, varies. Tho "aneurysm" may be a mere pouching of the aortic wall, or a sac as large as a baby's head. The damage to tho cusps may be trifling or extreme. The coronary narrowing may be slight or complete.

None of these lesions necessarily produce symptoms. One of our patients with a large aneurysm and well-marked aortio yalvular disease had no cardiac symptoms. Ho was 
admitted into hospital on account of a chill, which followed a very gallant and successful attempt to save a comrade who had fallen overboard in wintry weather. He had carried on his work easily and well. Another patient, a labourer, was admitted on account of the thrombosis of a popliteal aneurysm. He had well-marked aortic valvular disease, which had never inconvenienced him. A padre, who had had an infarct of his heart in 1920; carried on his work, easily and well, for more than five years after his convalescence, and even took on fresh responsibilities, though on his death in 1928 a large area of fibrosis was found to be present on the posterior wall of the left ventricle and the adjacent septum.

The symptoms of syphilitic heart disease do not differ from those of similar disease due to other causes. But although the symptoms of rheumatic and syphilitic disease are essentially similar there are some features peculiar to each. The dyspnoea of rheumatic disease is most often associated with exertion, while that of the syphilitic form often occurs in paroxysms at night, when the patient is in bed. One of our patients, who admitted that he had been becoming more short of breath on exertion for a few months, but had paid no attention to his discomforts, was forced to seek help when, without warning, he was wakened from sleep by paroxysms of intense breathlessness. Pain, too, may be of this paroxysmal character, and occur at night, without warning. These paroxysmal attacks are, of course, due to coronary obstruction, which is much more rare in the rheumatic disease.

Continued pain is sometimes due to aneurysm. In rheumatic disease continued pain is usually due to pericarditis or infarctions. The palpitation of rheumatic disease is generally due to an alteration of the cardiac rhythm, extra-systoles, fibrillation, etc., while in syphilitic disease it is usually the result of gross incompetence of the aortic valve and the throbbing of the arteries. If the aorta is dilated the palpitation may be referred to the base of the heart rather than to the cardiac area generally. Oedema in rheumatic disease is of gravity distribution, while in syphilis it may be local, from pressure of an aneurysm upon a vein. Complaint of constriction, oppression, or discomfort over the manubrium on exertion is suggestive of aortitis.

The appearance of the patient may be instructive. The rheumatic patient may be cyanosed, or, occasionally, pale, if an acute endocarditis has supervened. The syphilitic patient is often thin and emaciated, and perhaps of sallow, earthy complexion. Physical signs are sometimes suggestive. Not infrequently pulsation, from some slight dilatation of the vessels, is well marked over the upper part of the chest, above the sternum, or above the clavicle. A systolic aortic murmur sometimes precedes the advent of the diastolic murmur, though stenosis of the orifice is rare. The second aortic sound may be accentuated, though the blood pressure is not high, and may be intoned or ringing in character. The $x$-ray picture may show enlargement of the aortic shadow, even in minor dilatations, the normal outlines of the heart and aorta being lost.

The association of syphilis with aneurysm has long been recognized (Ambroise Paré6), but was forgotten by most writers until, in the latter half of last century, the connexion was again stressed by our army surgeons (Lewer, ${ }^{7}$ Lawson, ${ }^{8}$ Welch ${ }^{2}$, and in $1909^{10}$ spirochaetes were demonstrated in syphilitic aortitis. Further researches, using the Wassermann test, have emphasized the frequency of the connexion In our series of 66 cases of chronic aneurysm, 49 (74.2 per cent.) were syphilitic.

The incidence of aneurysm points the same moral. It is most common in men between 40 and 60 , the age when syphilitic disease of the heaitt is most frequent; our youngest patient was aged 26 . Usually the condition occurs ten to twenty years after the primary infection, but it has been reported during the secondary stages (Lewer, ten months after the chancre), and in one of Osler's cerebral cases eighteen months after infection.

Chronic Aortic Valvular Disease.

Syphilis is an important etiological factor in chronic aortic valvular disease. In a clinical series of 224 cases it was apparently causal in nearly a third of the cases.
The incidence in men and women, however, differs, as it occurred in rather more than a third of the men, and rather less than a fifth of the women. The age incidence is important. Nearly half of the cases in the forties and the fifties were due to syphilis, while only one case occurred under 30 , and less than a quarter of the patients over 60 were syphilitic. In the post-mortem series of 145 cases rather less than a fifth of the cases wero syphilitic. Nearly a fourth of the patients in the forties and fifties were syphilitic, while after 60 the incidence diminished to less than an eleventh.

It is interesting to note that in the clinical series the incidence of syphilis and rheumatism is approximately the same-32.1 and 33.9 per cent. The age and the sex incidence, however, differ. Less than a third of the men and more than a half of the women were rheumatic, and nearly a third of these patients were under 30 years old. The occurrence of aortic valvular disease in the forties or the fifties, especialy in men, without a clear history of antecedent rheumatism, is thus very suggestive of a specifio origin of the lesion.

The physical signs of aortic valvular disease are merely the signs of the valvular lesion, irrespective of its origin. It has been suggested that the absence of the second aortic sound suggests syphilis rather than rheumatism. It is quite true that the second sound usually persists in the rheumatic group; it is often accentuated, and frequently intoned in the degenerative cases, as one would expect from the nature of the lesions. Too much attention, however, must not be paid to this point, as it is really more an index of the degree of the lesion than a suggestion of its cause; but attention should be paid to the second pulmonary sound.

In syphilitic disease of the aortic valve the mitral valve is rarely affected, while in the rheumatic disease the mitral valve is affected in the majority of cases. In a clinical series of 51 syphilitic cases the aortic valve was alone involved in 31 cases; and in a series of 146 cases of acute endocarditis in 18 cases alone. The post-mortem series are equally striking. The mitral valve was only affected in one of 27 syphilitic cases, but in 45 -more than 25 per cent. -of 163 cases in the non-syphilitic group. In mitral disease the second pulmonary sound is usually accentuated, and its presence thus suggests coincident mitral disease, and so a rheumatic origin of the double lesion.

Aortic aneurysm and dilatation of the aorta are usually due to syphilis. Widespread pulsation over the upper part of the chest, pulsation in the suprasternal notch, and dilated carotid arteries thus suggest a syphilitic cause. In some cases an abnormal distribution of the murmurs, beyond the cardiac area, points to a dilatation of the aorta which has not yet shown itself by the signs which we have just mentioned. All these data, however, may be absent, and the diagnosis will depend upon the recognition of a specific infection in the past, a positive Wassermann reaction, or syphilitic stigmata elsewhere.

\section{Chronic Mitral Valvular Disease.}

Mitral valvular disease is rarely due to syphilis. In a clinical series of 102 cases of chronic mitral disease only two patients were known to be syphilitic, and in another series of 121 cases only one patient. In the post-mortem series only one of the 60 syphilitic patients had mitral disease, in this case coincident with aortic disease. In the mon-syphilitic group (330 cases) there were 55 cases of coincident mitral and aortic disease, as well as 32 cases in which the mitral valve alone was affected.

The incidence of acute endocarditis, too, differs. Only 5 of the 27 syphilitic aortic. cases were accompanied by acute endocarditis; while 35 of the 55 cases of aortic and mitral disease, and 16 of the 32 purely mitral cases, presented some degree of acute endocarditis at necropsy. It is, of course, well known that patients suffering from a chronic rheumatic endocarditis frequently develop an acute reinfection of the valve affected.

The sex and age incidence of aortic and mitral discase differ. In the clinical series of aortic disease 173 were male and 51 female. In the post-mortem series 112 were male and 41 female. The combined aortic and mitral series show 31 males and 24 females. The pure mitral 
cases show 14 males and 18 females. In the clinical aortic series 63 of 224 cases occurred before the age of 40 , and in the post-mortem series 15 of 153 cases. In the mitral group 13 of the 32 cases, and in the combined group 23 of the 55 cases, occurred before this age.

\section{Jlyocardial Disease.}

We have tried to estimate the relative frequency of syphilitic disease of the myocardium by examination of our data. The clinical scries, however, affords little information, for the majority of the patients were unable to give a full account of their past illnesses or, indeed, to permit a complete examination. But it is interesting to note that the maximal incidence occurred in the fifties and the sixties, a decade later than the maximal incidence of the syphilitic groul in the post-mortem series. In the latter series disease of the coronary arteries was more frequent in the syptilitic cases ( 23.3 per cent.) than in the nonsyphilitic cases (15.7 per cent.). The incidence of fibrosis in the two groups, howerer, is but little different (50 per cent. and 57 per cent.), which suggests that the degree of the arterial lesions is of more importance than its cause.

The incidence of fibrosis, with and without coronary disease, is greater in the non-syphilitic (21.2 per cent.) than in the stphilitic group (16.6 per cent.). This is probably due to the frequency of valvular disease in the non-coronary cases ( 16 of 40 ), and the resulting myocarditis.

We have been unable to discover any symptoms which are suggestive of a specific origin of myocardial disease; but the occurrence of symptoms of myocardial weakness without obvious cause should at once suggest the possibility of a syphilitic origin, particularly if the patient has not reached the age of 60 . The majority of the patients suffering from gumma of the heart seem to have been "admitted dead" into hospital."

\section{Angina Pectoris.}

The connexion between syphilis and angina has been stressed by many writers, but the exact relationship has not yet been determined, as the cause of angina is still disputed. The current theory, a revival of one first suggested by Allan Burns ${ }^{2}$ in 1809, has been elaborated from the experimental side by J. A. MacWilliam ${ }^{13}$ in 1923 , and from the clinical side by $C$. S. Kcefer and W. $H$. Resnik $^{14}$ in 1928. According to this theory angina is due to anoxaemia of the heart muscle, most commonly as the result of disease of the coronary arteries. The conception is physiological, not anatomical-a relative insufficiency of the blood supply of the heart, taking into account the work which the heart is accomplishing at the moment.

We cannot find many statistics as to the incidence of syphilis in angina. Huchard ${ }^{15}$ recognized a syphilitic taint in 170 of 740 cases, and Josue ${ }^{18}$ found a positive Wassermann reaction in 33 per cent. of his patients. Heimann ${ }^{17}$ noted that 68 of his 105 cases of cardio-vascular syphilis made a complaint of pain, and in 44 pain was the cardinal symptom. In 3 of 7 cases in which we secured a postmortem examination a syphilitic aortitis was present.

Angina is most common between the ages of 50 and 70 . This is a decade later than the common age of aortitis as shown post mortem. But, although it is uncommon before 30 , it may occur earlier, as in Wild's patient aged 12, in Moxon's patient aged 11, and in Daniélopolu's syphilitic patient aged $20 .{ }^{18}$ There does not seem to be any special symptom suggestive of a syphilitic origin. The occurrence of angina before the age of 40 , and its association with aortic valvular disease, should suggest a syphilitic cause, and an investigation into the possibility of a syphilitic origin should be made.

\section{Diagnosis.}

The chief causes of heart disease are rheumatism, ayphilis, bacterial endocarditis, and the degenerative lesions, of which atheroma is the type. It is difficult to say which is the most important, as their activities are maximal at different periods of life. Rheumatism is most important in early life, the degenerative lesions towards its close. Bacterial endocarditis accompanies rheumatism, while syphilis enters the stage at the very prime of life. Their actions, too, differ. Rheumatism and bacicrial endo- carditis attack the valves and the pericardium. Degenerative lesions attack the valves and the arteries, and syphilis follows suit. The diagnosis of ayphilitic disease of the heart depends upon the recognition that syphilis is an important cause of heart disease.

In a general way syphilitic heart disease occurs in the later phases of the illness, when tho primary illness has been almost forgotten and specific symptoms are absent. It is most frequent in the forties and the fifties, and in men as contrasted with women. The symptoms it causes are those of the cardiac lesion, and not those of syphilis. Aortitis and aneurysm are so frequently due to syphilis that their mere recognition suggests the cause. Of the valvular lesions mitral disease is so seldom associated with syphilis that its influence can be ignored. Aortic valvular disease, on the other hand, is frequently due to syphilis, whose influence should always be investigated. Myocardial disease and angina pectoris are of ten the result of syphilis, but less frequently than aortic valvular disease. Pericarditis is rarely due to syphilis.

The personal and the family history are important, but these are not always accurate. One must remember that the primary manifestations are sometimes trivial and unrecognized, even when suspected. The crror may be due to the patient or to his medical adviser. A positive Wassermann reaction is important, for although the test is not specific in the serological sense, it is rarely obtained in this country save in syphilitic cases. $\boldsymbol{A}$ negative result has not the same significance, for the reaction is only positive in about 75 per cent. of patients in the tertiary stage, the period at which most syphilitic cardiac patients come under observation. We have all, we imagine, seen good results follow specific treatment in patients whose Wassermann reaction has been frankly negative.

A full routine examination may discover signs of diagnostic significance elsewhere than in the heart. Scars on the penis, the throat, or the shins, nodes on the bones, loss of the deep reflexes or the light response in the eyes, leucoplakia, orchitis, unequal pupils, iritis, choroiditis, periphlebitis of the retinal vessels, optic atrophy, or the early symptoms of tabes or general paralysis of the insane, if present, may be decisive.

\section{Treatment.}

In the treatment of cardiac syphilis the two sides of the question-the constitutional and the local-must be borne in mind. From the cardiac side the treatment is the same as in the non-syphilitic varieties. From the constitutional side specific treatment is required as urgently as in syphilitic disease elsewhere. Too much, however, must not be expected from such medication, for the lesions, when recognized, are as a rule in an advanced stage.

The most common lesion is endarteritis, which is often accompanied by thrombosis. But the latter, as Gowers pointed out many years ago, is not a specific prccess, and is not amenable to specific treatment. The necrotic tissue cannot be revived; but at its periphery the tissues are linked up with other arterial territories, and if, by specific treatment, the lumen of their arteries can be enlarged the nutrition of the tissues, starved but not killed by the cessation of the blood supply from the thrombosed ressel, will be increased, and their functional activity correspondingly increased.

Syphilitic lesions may be "active" many rears after the initial infection. One of our patients, who died at the age of 50 , had contracted the disease thirty-three years before. The aorta, which presented syphilitic scars over most of its thoracic course, showed one small typical " active" nodule.

The nature of the specific treatment requires concideration. Should a "sterilizing" treatment be attempted or a milder course pursued? Are the arsenical pieparations advisable, or mercury and iodide? It is obviously inadvisable to administer full doses of salvarsan to a man racked with pain or distressed with orthopnoea. Renal disease, which is so often present, is another contraindication, and, of course, massivo oedema; but is it inadvisable in the case of a strong man whose disability has been discovered in the course of some routine examination, in whom no symptoms of cardiac difficulty obtain, or in those whose disabilities are slight? 
Those of us who had to deal with malaria during the late war found a similar difficulty before us. But it was soon apparent to us all that, although we could treat primary malaria successfully by massive doses of quinine, such treatment was useless, if not harmful, in the chronic infections. Smaller doses were more efficacious. Syphilitic heart disease is of the chronic type.

Another consideration should also be borne in mind. It is well known that the administration of tuberculin in large doses to a patient suffering from phthisis may produce both a general reaction and a local one at the site of the focus. A similar reaction may occur in syphilis if large doses of the arsenical preparations are administered (Jarisch-Herxheimer reaction). ${ }^{19}$ The general opinion is that this is due to the sudden liberation of toxins from the bodies of the organisms that are destroyed. But, whatever the cause, it is obviously hazardous to expose a patient with, possibly, a narrowed coronary orifice to the danger of a local reaction and the complete obstruction of the artery. If arsenic is used in such cases it should only be subsequent to a course of mercury and iodide, and in moderate doses.

We have thus continued to treat cases of cardiac syphilis along the usual lines, with moderate doses of mercury and iodide, etc., orer long periods of time. Small doses of kharsivan, or neokharsivan, are sometimes useful, as are Donovan's solution, the green iodide of mercury, arsenic iodide, and potassium iodide. The results which we have observed have sometimes been extremely satisfactory. Wo must always remember, howerer, that the truc treatment of cardiac syphilis is preventive, the successful treatment of the primary illness, and that cardiac syphilis is the result of error, on the part of the patient or his medical adviser.

REFERENCES.

1 Osler and Macrae: Modern Medicine, 1925, ii, 390.

3 Official History of the War. Diseases of the War, 1923, ii.

4 G. A. Allan: Glasgow Med. Journ., August, 1924.

5 Robert Muir : Textbonk of Pathology, 1929, 274.

6 Ambroise Paré, London, 1634, 287.

A. Lewer : Army Medical Report8, 1852, 512

8 Inspector General Lawson:.Army Medical Reports, 1868, app., 267.

9 F. H. Welch : Diseases of the Heart among Soldiers, 1870.

10 J. H. Wright: Boston Med. and Surg. Journ., 1909.

$" \dot{W}$. P. Herringham : St. Bartholomew's Hospital Reports, 1903, xxxix, 9.

12 Alian Burns: Observations on Some of the Nlost lmportant Discases of

the Heart, Edin., 1809, 138

3 J. A. MacWilliam: British Medical Journal, 1923, i, p. 51 ; ii, pp. 215,

14 C. S. Keefer and W. H. Resnik : Arch. Int. Med., 1928, xli, 769.

$15 \mathrm{Fi}$. Huchard: Maladies du cour, 1899, ii, 54.

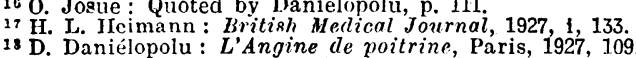

13 D. Daniélopolu: L'Angine de poitrine, Paris, 1927, 109.

\section{THE DIAGNOSIS OF FRONTAL TUMOURS.* BX}

JAMES COLLIER, M.D., F.R.C.P.,

CONSULTING PHYSICIAN AND EMERITUS LECTURER ON NELROLOGY, ST. GEORGE'S HOSPITAL, LONDON.

From the very nature of the cerebral hemispheres the rogional diagnosis of disease situated therein is often a matter of peculiar difficulty. The cerebral hemispheres have their origin as appendages to the nervous mechanism subserving the olfactory sense, and havo doubtless acquired dominanco in the nervous system from the importance of the sense of smell in acquiring food and scenting danger, and so providing for the surviral and advance of the race.

The crebral hemispheres have no primary and direct connexions with any other function than that of smell, nor with any other region than the olfactory region. Secondary comnexions are dereloped, as evolution proceeds, by mediate paths with every function and with every rugion of the body; and in the higher vertebrates the cerebral hemispheres become increasingly and moro directly linked up with specific bodily functions, by the development of ocrtain great white tracts which directly impinge upon, or take their origin from, the corebral cortex. Interference with the integrity of these white tracts produces definite

- A paper read in opening a discussion in the Section of Neurology and Psychological Medicine at the Annual Meeting of the British Medical
Afsodation, Manchester, 1928. and recognizable clinical signs. Even in man there are not many of these paths. There is the visual path entering the cortex of the cuneus and posterior pole. There is the auditory path to the temporal regions, the higher sensory path to the parietal lobes, and paths subserving incoming and outgoing speech connected with the left temporal lobe. With the knowledge of the anatomical details of these paths and of the results of lesions interrupting them, our knowledge of cerebral localization of function begins and ends. It is only because quick and easy compensation does not occur for lesions of these paths in man that we havo even this slight knowlerlge of cerebral localization. In vertebrates lower than man such compensation occurs readily. Sherrington remored successively the right and left motor cortex from a chimpanzee. The animal recovered his motility perfectly. And when long recovered and a normal chimpanzee, an investigation was mado to find where the motor cortex had been transferred to, and it was found that there was no excitable cortex-that thero was no motor area in this perfect animal. No local motor function of the cortex liad been abrogated by the destruction of the motor cortex. Only the pyramidal path had been destroyed, and Nature had found another way out for the exteriorization of motor function.

Hughlings Jackson, in the Croonian Iectures for 1884, formulated a theory of the way in which the nervous system worked. It has long since been proved to bo anatomically acceptablo by Golgi and his followers, who built up the "nenrone", concept. It scems to be increasingly acceptable, physiologically and clinically, as time goes on. That part of Jackson's theory which concerns us on this subject may be put in two sentences. There are several structural and functional levels in the nervous system from the lowest to the highest. In the lowest level, which is represented by the spinal segment, simple structure and simple function aro directly represented, but with each higher level everything below is re-represented in increasing compound proportion, so that in the highest level there can be no local representation, but a representation of some attribute of the physiological individual as a whole. Hughlings Jackson-so often on the lips of nourologists, so little read and so ill understood -is often called the "father of cortical localization," for his theory preceded Hitzig's experimental work; ho was, in fact, the destroyer of cortical localization in that his theory is directly antagonistic to local residence of function in the highest part of the nervous system. Therefore, in considering what may bo the signs of tumours within tho frontal lobe, if we are to adrance in knowledge we must divest our minds of any idea of local function, mental or other, peculiar to lesions of the prefrontal lobes; we must expect a varying clinical picture, and of ten ono which is negative so far as localizing symptoms aro concerned. Wo must bear in mind the great facility for compensation for local lesions within this region so fully emphasized by Gowers forty years ago, and keep in mind the disturbing effects of long-standing increase of intracranial pressure and of hydrocephalus upon the value of those signs which we use for localization, and strive to make a collect of those signs which have been useful to us collectively in the successful localization of tumours within the frontal lobe, for we do locate them successfully very often. I may conveniently divide the signs which are used for the location of a tumour within the prefrontal lobes into five categories :

1. The negative signs.

2. The contiguous signs.

3. The signs which have been deemed peculiar to, or especially marked in, prefrontal lesions.

4. The signs peculiar to lesions within the anterior fossa of the skull.

5. The signs revealed by ventriculography.

Negative Signs.

The negative signs are of especial importance, since tumours within the posterior fossa below the tentorium, and those of the occipital, parietal, central, and left temporal regions, except sometimes in their latest stages, give unmistakable signs of their position. Therefore if there be no definite localizing sign, it is commonly a matter of a 